\title{
Evaluation of Variable Pitot Inlet Concepts for Transonic and Supersonic Civil Aviation
}

\author{
Stefan Kazula ${ }^{1, *}$, Marcel Mischke ${ }^{1}$, Paul König ${ }^{1}$, and Klaus Höschler ${ }^{1}$ \\ ${ }^{1}$ Brandenburg University of Technology, Chair of Aero Engine Design, 03046 Cottbus, Germany
}

\begin{abstract}
This paper describes the selection of the ideal variable inlet concept group by utilising results of aerodynamic investigations, system safety analyses and integration studies. Aerodynamic and functional inlet requirements are explained, and variable inlet concept groups are introduced. The concept evaluation by means of a weighted point rating is presented. The respective concept groups are analysed and evaluated regarding economic, functional and safety requirements. By means of this evaluation, the concept group that adjusts the inlet geometry by rigid segment repositioning is identified as most suitable concept group. The early selection of the most suitable concept group enables more detailed subsequent concept investigations, potentially enabling the technology of variable inlets for future commercial aircraft.
\end{abstract}

\section{Introduction}

Variable pitot inlets have the potential to reduce aircraft drag, allowing for increased efficiency and faster flight speed compared to aircraft with rigid inlets [1], [2]. In contrast to 2-dimensional variable inlets, which are utilised in current supersonic applications, circular variable pitot inlets can provide an air flow of higher uniformity to the aero engine's compressor system. Thus, variable pitot inlets could allow for a shorter length and thus decreased weight, potentially enabling supersonic commercial aviation up to Mach 1.6.

Several research studies concerning circular variable inlets with adjustable lip and duct geometry have been conducted [3], [4]. However, this technology has not made its way into service yet. A potential lack of safety and reliability is one explanation for this, as the additional function 'adjustability' leads to an increased complexity of the inlet system [5].

Hence, within the scope of a feasibility study for variable inlet concepts, the methodological development process is combined with the safety assessment process in aviation according to Aerospace Recommended Practices ARP 4754A [5]. Among others, this process comprises

- the identification of ideal geometries for variable inlets concerning drag and flow uniformity for flight speeds up to Mach 1.6 and their potential range benefit [6],

- the development of inlet concept groups that adjust the geometry by rigid segment repositioning, by elastic surface deformation or by boundary layer control [7],

- the application of the safety methods Functional Hazard Assessment (FHA), Fault Tree Analysis (FTA) and Common Cause Analysis (CCA) [8], [9],

- the integration of ice detection and protection systems for variable inlet concepts [10]

* Corresponding author: Stefan.Kazula@b-tu.de

(C) The Authors, published by EDP Sciences. This is an open access article distributed under the terms of the Creative Commons Attribution License 4.0 (http://creativecommons.org/licenses/by/4.0/). 
- $\quad$ as well as subsequent design adaptations.

This paper describes the selection of the ideal group of variable inlet concepts by utilising results of aerodynamic investigations, system safety analyses and integration studies. First, the tasks and requirements of inlets, the trade-off in inlet design, as well as the variable inlet concept groups are presented. Afterwards, the concept evaluation methodology and evaluation criteria are selected and presented. Subsequently, the respective concept groups are analysed and evaluated regarding economic, functional and safety requirements, allowing for the identification of the most suitable group of concepts for variable inlets. The selection of the most suitable concept group at an early stage of the product development enables a more detailed investigation during later phases of the development process, potentially leading to an improved safety and operating efficiency of the resulting variable inlet concept. This could enable the technology of variable inlets for commercial aviation.

\section{Variable aero engine inlets}

\subsection{Inlet requirements}

An aero engine requires air to produce thrust for the aircraft. The air is provided by the nacelle inlet, also called intake. The primary objective of the inlet is to divide the free stream in front of the aero engine at the stagnation point depending on the operating conditions into an internal and an external air flow, while avoiding flow separation and minimising aerodynamic drag [11].

The inlet must meet these aerodynamic requirements that lead to opposing ideal geometries to ensure optimum efficiency and reliable operation [12]. Flow separations and potentially resulting hazardous events during take-off and climb operation up to Mach 0.3 need to be avoided by a thick and round inlet lip, which results in increased drag, especially during cruise operation [13]. High efficiency at fast subsonic cruise flight above Mach 0.8 can be obtained by using a thinner lip contour combined with a curved and long forebody, resulting in minimised drag [14], [6]. For supersonic flight up to Mach 1.6, pitot inlets with sharp inlet lips and long flat forebodies can be applied without significant aerodynamic losses [14]. At low aircraft velocities during take-off and climb, where high angles of incidence and crosswind can occur, sharp or thin inlet lips are sensitive to flow separation and its potential negative effects [14].

Hence, conventional rigid subsonic inlets can only accomplish a geometry that provides a trade-off concerning minimum drag at high velocities and avoidance of flow separation at low velocities. The limitations of conventional rigid inlets can be circumvented by using variable pitot inlets that adjust the ideal inlet geometry for each flight condition. Thus, a variable pitot inlet could provide geometries that are efficient and reliable during take-off, climb as well as subsonic and supersonic cruise conditions. According geometries have been identified by Kazula et al. [6].

While the inlet weight must be minimised to achieve maximum flight range, the inlet still should be resilient to foreign object damage, sand erosion, hail and bird strikes. Furthermore, it is necessary that thrust can be maintained to a certain level after a single bird strike to assure that the flight can continue safely [15]. Furthermore, the inlet must protect itself and the compressor system from ice accretion and its potentially hazardous consequences, such as impact damage and flow separation. Hence, an ice protection system, usually, an electrothermal or a bleed air system, is integrated in the inlet to avoid icing of the inlet lip [16]. Additionally, probes for pressure and temperature measurement in front of the fan can be part of the inlet. Moreover, the inlet has to reduce fan and compressor-related noise emissions, usually by integrating acoustic treatment into the diffuser wall. 


\subsection{Variable inlet concept groups}

\subsubsection{Rigid segment repositioning}

The group that adjusts the inlet geometry by repositioning of rigid segments varies the inlet lip, the entry area and potentially the inlet length through axial motion, e.g. by an electric spindle drive. The adjustment of the inlet length can result in improved aerodynamic properties, but also in significantly increased complexity of the design.

The variation of the inlet entry area is enabled by segmenting the inlet over its circumference in circular segments [10]. The circular segments can be equipped with fixed rigid sealing elements, which form a part of the inlet surface during take-off. During cruise flight, the sealing elements of adjacent circular segments overlap each other, and thus enable a reduction of the cross-sectional area. Further options for the sealing element design are elastic sealing elements, rotating circular segments, and separate sealing elements between the circular segments.

Safety analyses [9] underline the importance of ice protection for this concept group and suggest an extension of the protected surface area to avoid ice formations between the circular segments, resulting in increased power requirements of the ice protection system [10]. Interactions between components within the inlet zone, as well as zones in the vicinity must be mitigated, for instance between the ice protection system and the adjustment system. Leakage of any fluid in proximity to electrical equipment of the adjustment system can cause damage and hazards, which are avoided by design adaptations, e.g. over-pressure breakout panels, ventilation, drainage and heat protection for the adjusting system [8], [9]. Due to the physical adjustment of the inlet geometry, a flexible design of wiring and pipe installation to minimise stresses, as well as a minimum clearance between those components to avoid friction and collision are required [9].

Some functions of the adjustment system are similar to functions of certain ice protection systems, potentially allowing for fewer subsystems and resulting in reduced weight, as synergies could be utilised [10].

\subsubsection{Elastic surface deformation}

The concept group that adjusts the inlet geometry by deformation of elastic surface material can vary the inlet lip, the entry area, and the length. The main advantage of this group is the step-less surface, as the surface can be made of one piece. The limitations of this concept group are the necessity of a supporting structure to ensure that the geometry remains in the desired state and the reduced lifetime of the elastic surface material. Smart materials, elastomers and elastic composites could be utilised as elastic surface material [17], [18], [19]. Concepts of this group can vary the inlet lip, the entry area and the length through axial motion of an electric spindle drive and deformation of the flexible surface material, while the supporting structure ensures that the geometry remains in the desired shape [10].

The inlet lip of this concept group should be made of aluminium to withstand external loads. As conventional rigid acoustic liners would prevent the contour adjustment, either acoustic liners with flexible walls [20] or sound cancellation by counter-noise [21] should be considered as noise attenuation options. A potential ice protection system for this inlet concept group must cover approximately the same area as for a conventional rigid pitot inlet, which usually only protects the inlet lip. Furthermore, the ice protection system must not interfere with the adjustment system or the elastic material, for instance by collisions, leaking fluids or temperatures. Due to the physical adjustment of the inlet geometry, the design of the wiring must be flexible, and the pipe installation must be telescopic.

\subsubsection{Boundary layer control}

The concept group of boundary layer controlling inlets avoids flow separations during takeoff, while using a rigid conventional inlet design with a geometry that is ideal for cruise 
flight. These concepts have a high potential for aerodynamic efficiency, as they can be designed without steps and gaps on the surface, while producing the lowest possible drag during cruise conditions. The main advantage of boundary layer controlling inlets compared to other concept groups is that weight could be decreased, and reliability increased, as no complex adjustment system is required. Furthermore, conventional acoustic treatment and ice protection could be utilised. However, the much thinner inlet geometry still must withstand bird strikes to a certain degree. Furthermore, fail safe design principles, e.g. redundancy, must be included to avoid flow separations in case of a system failure at low aircraft velocities. Boundary layer separation in the inlet region can be prevented by

- elimination of the velocity difference between wall and air flow,

- suction of the delayed boundary layer into the inside of the body by slots in the body wall, - tangential blowing into the boundary layer [22], [23].

By moving the wall in flow direction, the velocity difference between wall and flow is eliminated and boundary layer formation can be prevented. This principle is a very effective way to avoid boundary layer separation [22]. However, it is not applicable to variable inlets.

Boundary layer suction has been utilised successfully on aircraft wings and even on supersonic inlets to reduce the boundary layer thickness [24], [22]. Inlets could also prevent flow separation by suction of the delayed boundary layer into the inside of the inlet through activatable suction slots. The suction slots can be integrated in the lip, the outer planking and the inner diffuser wall and must be distributed over the circumference of the inlet. An ideal distribution of the slots should ensure a highly uniform inlet flow, while requiring as few suction slots as possible. The suction slot opening and closing must be reliably performed by valves or flaps, as a malfunction of separate suction slots can lead to nonuniform flow to the compressor system and ultimately result in hazardous events. Hence, the suction slots must be protected from dirt, moisture and foreign object damage, but also from icing and its consequences.

Tangential blowing of air through surface slots into the boundary layer, can prevent boundary detachment, by supplying kinetic energy. As with the suction slot concept, tangential blowing enables the utilisation of a cruise flight optimised geometry, while ensuring reliable operation during take-off and climb. Conventional acoustic treatment and ice protection solutions can be applied here as well. The blowing slots require valves or flaps. The most challenging aspect of this concept is the selection of the correct air quantity, velocity, pressure and temperature at high uniformity for each flight condition. Minor mistakes in this selection may cause flow separations or damage to the compressor system due to over-temperature, potentially leading to hazardous events. Hence, protection of the blowing slots from icing and other influences is of importance [10].

\section{Evaluation}

Various evaluation methods for technical products with a wide range of complexity and for different decision tasks exist. These methods support the decision-making process in an objective and comprehensible way when selecting from several potential solution options [25]. This feasibility study uses a weighted point rating to rank the variable inlet concept groups. In the weighted point rating, the identified criteria are weighted according to their significance to the success of the product.

\subsection{Criteria identification}

For the variable inlet concept groups, the most important evaluation criteria can be derived from the requirements document. These criteria are aerodynamic efficiency, ease of 
integration, weight, safety, reliability and life cycle costs, as well as development and manufacturing costs.

\subsubsection{Aerodynamic efficiency}

The aerodynamic efficiency of a variable inlet is primarily determined by the geometries that can be set by the variable system. Deviations from the ideal inlet geometry can occur in the field of circularity, diameter adjustability or lip thickness. Furthermore, steps, gaps and surface quality can influence the aerodynamic properties of the inlet.

\subsubsection{Ease of integration}

The ease of integration of a system is defined by the required installation space of the system components, as well as interfaces and interactions with components within the inlet zone, e.g. probes, acoustic treatment or a potential adjustment system, and in the vicinity of the inlet. These interactions between components within the inlet zones, as well as zones in the vicinity are investigated best by conduction of a Zonal Safety Analysis (ZSA) [9].

\subsubsection{Weight}

The weight of a variable inlet is driven by the density and strength properties of the utilised material, and by the additionally required system components, e.g. actuators, pipes and wiring, by the operating resources, as well as by potentially necessary safety adjustments, e.g. bulkheads, grounding and breakout panels.

\subsubsection{Safety}

The adjustability of the variable inlet must not adversely affect its primary function of supplying air to the engine. Hence, no hazardous events should occur due to the variable inlet or a malfunction of that system. Potential safety hazards are flow separations, damage to components in the proximity and fire. Flow separations could occur due to an unsuitable geometry at transient conditions. Damage to components in the proximity can be caused by spalling ice during icing conditions or by inlet components after a bird strike. Fires could result from combinations of hot surfaces and leaking flammable fluids.

\subsubsection{Reliability and life cycle costs}

Service life, reliability and life cycle costs of variable inlet concepts are closely associated. Reliability is mostly based on the number of parts and the ability to withstand erosion, fatigue and limit loads. Furthermore, moisture can lead to corrosion, sand and dirt can block holes and valves, and friction between moving parts can impede movement or damage parts. Moreover, chemicals, e.g. anti-icing fluids, can influence the lifetime of the utilised materials. The life cycle costs of variable inlets are additionally influenced by its power requirements, necessary consumables, e.g. lubricants, as well as maintenance intervals and efforts.

\subsubsection{Development and manufacturing costs}

The development costs of a variable inlet system are mostly based on the experience with the utilised technologies and materials in the field of aviation. If little experience concerning the geometry adjustment system and the utilised materials exists, more analyses and tests are required. These lead to increased development time and therefore costs. Moreover, innovative materials, special manufacturing methods, additional quality controls, as well as increased required assembly and transport efforts can result in higher manufacturing costs.

\subsection{Criteria weighting}

The weighting of the criteria is obtained by pairwise comparison. Hereby, all criteria are cross-checked in pairs by the evaluation group [25]. After all criteria have been compared 
with each other, each criterion $i$ has a total value $p_{i}$, which is the sum of the values in the respective row in Table 1. Dividing this row sum $p_{i}$ by the sum of all assigned cell values $P$ returns the relative weighting factor $w_{i}$ of a criterion [25]:

Table 1. Criteria weighting via pairwise comparison

\begin{tabular}{|c|c|c|c|c|c|c|c|}
\hline $\begin{array}{l}\text { Cell values } \\
2=\text { row criterion is more important } \\
\text { than column criterion } \\
1=\text { both criteria are equally important } \\
0=\text { row criterion is less important } \\
\text { than column criterion }\end{array}$ & 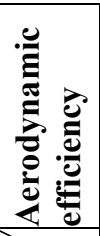 & 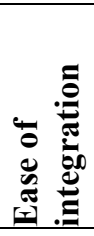 & $\frac{5}{300}$ & 莺 & 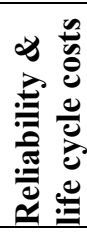 & 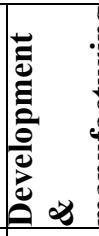 & 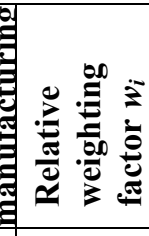 \\
\hline Aerodynamic efficiency & & 2 & 2 & 1 & 1 & 2 & 0.267 \\
\hline Ease of integration & 0 & & 1 & 0 & 0 & 1 & 0.067 \\
\hline Weight & 0 & 1 & 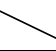 & 0 & 1 & 2 & 0.133 \\
\hline Safety & 1 & 2 & 2 & 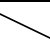 & 1 & 2 & 0.267 \\
\hline Reliability \& life cycle costs & 1 & 2 & 1 & 1 & & 1 & 0.200 \\
\hline Development \& manufacturing & 0 & 1 & 0 & 0 & 1 & & 0.067 \\
\hline
\end{tabular}

\subsection{Concept group rating}

For each concept group $j$, a numerical measure $m_{i, j}$ is assigned corresponding to the fulfilment of a criterion $i$ by that concept group. The evaluation has been performed based on earlier conducted safety analyses [8], [9], integration studies [10] and aerodynamic investigations [2], [6]. The evaluation of the fulfilment of a criterion by a variable inlet concept group has been carried out by assigning a numerical measure $m_{i, j}$ of 4 for very good fulfilment, 3 for good fulfilment, 2 for average fulfilment, 1 for bad fulfilment and 1 for very bad fulfilment. The repositioning of rigid segments concept group is the most suitable concept group, when not considering the relative criteria weighting $w_{i}$, compare Fig. 1.
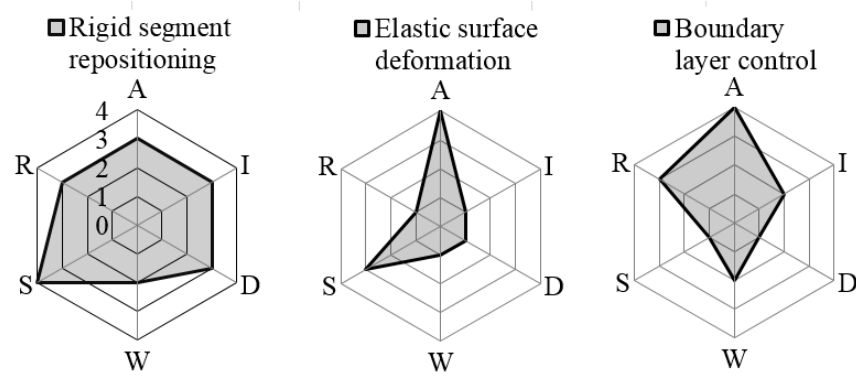

\begin{tabular}{|l|}
\hline $\mathrm{A}-\begin{array}{l}\text { Aerodynamic } \\
\text { efficiency }\end{array}$ \\
\hline $\mathrm{I}-$ Ease of integration \\
\hline $\mathrm{D}-\begin{array}{l}\text { Development and } \\
\text { production costs }\end{array}$ \\
\hline $\mathrm{W}-$ Weight \\
\hline $\mathrm{S}-$ Safety \\
\hline $\mathrm{R}-\begin{array}{l}\text { Reliability and } \\
\text { life cycle costs }\end{array}$ \\
\hline
\end{tabular}

Fig. 1. Illustration of the inlet concept group evaluation results

While the other concept groups can provide ideal geometries with perfect surfaces, the concept group that adjust the inlet geometry by repositioning of rigid segments has minor limitations concerning aerodynamic efficiency. These limitations arise from the small steps and gaps between the segments, the not ideal circular geometry and the constraints concerning entry diameter adjustment.

Apart from the adjustment system, the extended ice protection area and the number of parts, this concept group is much easier to integrate into the inlet than the other options. The elastic surface material concept group requires a complex internal supporting structure, innovative ice protection and noise treatment solutions, as well as an adjustment system. Furthermore, the influence of the ambient conditions on the surface material must be 
investigated. The boundary layer control concepts demand for pumps, pipes and many slots that must be protected from ice and dirt.

These components entail additional weight. The rigid segment and the elastic surface concept groups require adjustment systems, with the latter furthermore requiring a heavy supporting structure.

In case of a malfunction rigid segment repositioning concepts can automatically set a failsafe geometry. The additional risk of fire due to the adjustment system can be handled by design precautions, e.g. bulkheads [9]. The same applies for most elastic surface concepts. However, these concepts are more prone to material failures due to foreign objects or bird strikes, resulting in potential hazardous events. The boundary layer control concept group uses a thin geometry that is sensitive for flow separation during take-off and climb conditions, hence, a malfunction of the system can result in hazardous events.

While the high part number, the limited service life of the flexible wiring, the power and maintenance requirements of the adjustment system, as well as friction between the circular segments must be considered, rigid segment concepts provide long life and high reliability. The surface material of the elastic surface concept group has highly limited service life due to erosion and sensitivity to ambient conditions, resulting in short maintenance intervals with high efforts. Apart from the large number of suction or blowing slot and the associated maintenance and pump power requirements, boundary layer controlling concepts are reliable with low operational costs.

Rigid segment and boundary layer controlling concepts have low manufacturing costs, while the elastic surface material of the corresponding concept group could lead to high costs. Additional development efforts like simulations and tests would be required to certify elastic surface and boundary layer controlling concepts.

The relative rating represents the overall suitability of a concept group by considering the relative weighting. The relative rating is calculated from the sum of products of all numerical measures $m_{i, j}$ multiplied by the relative weighting factor of the respective criteria $w_{i}$, divided by the scale size 4 [25]. The rigid segment repositioning inlet concept group is the most suitable concept group with a relative rating $r_{i}$ of 0.783 , followed by the boundary layer control group with 0.633 and the elastic surface deformation group with 0.183 .

\section{Conclusions}

This paper describes the selection of the ideal concept group for variable pitot inlets by utilising results of aerodynamic investigations, system safety analyses and integration studies. Inlet requirements, the trade-off in inlet design, as well as variable inlet concept groups that adjust the geometry by rigid segment repositioning, by elastic surface deformation or by boundary layer control are described. Afterwards, the concept evaluation by a weighted point rating is presented. The respective concept groups are analysed and evaluated regarding economic, functional and safety requirements, allowing for the identification of the rigid segment repositioning group as the most suitable concept group for variable inlets.

The selection of the most suitable concept group at an early stage of the product development enables a more detailed design during later phases of the safe design process. This can potentially lead to an improved safety and operating efficiency of the final variable inlet concept. Furthermore, the remaining range benefit after considering additional weight, complexity and potential aerodynamic steps and gaps of the variable system can be determined. This way, the technology of variable pitot inlets for supersonic transport aircraft could be enabled by the safe design approach and be a way to achieve the ambitious ecological, safety and economic goals for future aviation. 


\section{References}

[1] F. Majić, G. Efraimsson, and C. J. O'Reilly, "Potential improvement of aerodynamic performance by morphing the nacelle inlet," Aerospace Science and Technology, vol. 54, pp. 122-131, 2016.

[2] S. Kazula, M. Wöllner, D. Grasselt, and K. Höschler, "Ideal Geometries and Potential Benefit of Variable Pitot Inlets for Subsonic and Supersonic Business Aviation," Proceedings of the 8th Eucass, 2019.

[3] L. da Rocha-Schmidt, A. Hermanutz, and H. Baier, "Progress Towards Adaptive Aircraft Engine Nacelles," Proc. of the 29th Congress of the International Council of the Aeronautical Sciences, 2014.

[4] N. G. Ozdemir et al., "Morphing nacelle inlet lip with pneumatic actuators and a flexible nano composite sandwich panel," Smart Mater. Struct., vol. 24, no. 12, p. $125018,2015$.

[5] SAE Aerospace, ARP4754A. SAE International, 2010.

[6] S. Kazula, M. Wöllner, D. Grasselt, and K. Höschler, "Parametric design and aerodynamic analysis of circular variable aero engine inlets for transonic and supersonic civil aviation," Proc. of ISABE, 2019.

[7] S. Kazula and K. Höschler, "A systems engineering approach to variable intakes for civil aviation," Proceedings of the Institution of Mechanical Engineers, Part G: Journal of Aerospace Engineering, 095441001983690, 2019.

[8] S. Kazula, D. Grasselt, M. Mischke, and K. Höschler, "Preliminary safety assessment of circular variable nacelle inlet concepts for aero engines in civil aviation," in Proceedings of the 28th ESREL, CRC Press, 2018, pp. 2459-2467.

[9] S. Kazula, D. Grasselt, and K. Höschler, "Common Cause Analysis of Circular Variable Nacelle Inlet Concepts for Aero Engines in Civil Aviation," in Proceedings of the 6th International Conference on Integrity-Reliability-Failure :, 2018.

[10] S. Kazula and K. Höschler, "Ice detection and protection systems for circular variable nacelle inlet concepts," CEAS Aeronaut J (2019). DOI 10.1007/s13272-019-00413-1.

[11] J. D. Mattingly, Elements of Propulsion: Gas Turbines and Rockets. AIAA, 2006.

[12] J. Seddon and E. L. Goldsmith, Intake aerodynamics, AIAA, 1999.

[13] W. J. G. Bräunling, Flugzeugtriebwerke, Springer Vieweg, 2015.

[14] S. Farokhi, Aircraft propulsion. Wiley, 2014.

[15] R. Hedayati and M. Sadighi, Bird strike. Woodhead Publishing, 2016.

[16] Rolls-Royce plc., The jet engine. Wiley, 2015.

[17] G. Akhras, "Nano \& Smart NDE Systems - Applications in Aerospace and Perspectives," 4th International Symposium on NDT in Aerospace, 2012.

[18] S. Murugan and M. I. Friswell, "Morphing wing flexible skins with curvilinear fiber composites," Composite Structures, vol. 99, pp. 69-75, 2013.

[19] M. Kintscher and M. Wiedemann, "Investigation of Multi-material Laminates for Smart Droop Nose Devices," ICAS, 2014.

[20] M. Mischke, S. Kazula, and K. Höschler, "A Comparative Concept Study and Evaluation for New Broadband Noise Absorbing Acoustic Liner Concepts for Civil Aviation," Proceedings of Global Power and Propulsion Society, 2019.

[21] Virginia Tech Intellectual Properties Inc., "Active control of aircraft engine inlet noise using compact sound sources and distributed error sensors," US5355417A.

[22] H. Schlichting, K. Gersten, and E. Krause, Grenzschicht-Theorie, Springer, 2006.

[23] H. G. Lueders, Experimental Investigation of Advanced Concepts to Increase Turbine Blade Loading. Washington, D.C.: NASA Lewis Research Center, 1968.

[24] A. L. Braslow, A History of Suction-Type Laminar-Flow Control with Emphasis on Flight Research. Monograph in Aerospace History, No. 13, 1999. NASA, 1999.

[25] J. Feldhusen and K.-H. Grote, Pahl/Beitz Konstruktionslehre, Springer Vieweg, 2013. 